\title{
SOME RESULTS AND PROBLEMS CONCERNING CHORDAL PRINCIPAL CLUSTER SETS*
}

\section{F. BAGEMIHL}

To Professor Kiyoshi Noshiro on the occasion of his 60th birthday

Let $\Gamma$ be the unit circle and $D$ be the open unit disk in the complex plane, and denote the Riemann sphere by $\Omega$. By an arc at a point $\zeta \in \Gamma$ we mean a continuous curve $A: z=z(t) \quad(0 \leqq t<1)$ such that $|z(t)|<1$ for $0 \leqq t<1$ and $\lim _{t \rightarrow 1} z(t)=\zeta$. A terminal subarc of an $\operatorname{arc} A$ at $\zeta$ is a subarc of the form $z=z(t)$ $\left(t_{0} \leqq t<1\right)$, where $0 \leqq t_{0}<1$. Suppose that $f(z)$ is a meromorphic function in $D$. Then $A(f)$ denotes the set of asymptotic values of $f$; and if $\zeta \in \Gamma$, then $C(f, \zeta)$ means the cluster set of $f$ at $\zeta$ and $C_{\mathscr{A}}(f, \zeta)$ is the outer angular cluster set of $f$ at $\zeta$ (see [13]). The principal cluster set of $f$ at $\zeta$ is the set

$$
\Pi(f, \zeta)=\bigcap_{\Lambda} C_{\Lambda}(f, \zeta),
$$

where $\Lambda$ ranges over all arcs at $\zeta$. As is well known, this set is of importance, and was introduced some time ago, in connection with the theory of boundary correspondence under conformal mapping. More recently the set

$$
\Pi_{x}(f, \zeta)=\bigcap_{x} C_{x}(f, \zeta)
$$

where $X$ ranges over all chords of the unit circle at $\zeta$, has received attention, notably in the work of Meier [12], who has used this set, which we call the chordal principal cluster set of $f$ at $\zeta$, in the formulation of his topological analogue of Plessner's theorem.

Because of the significance of the chordal principal cluster set in this connection as well as others, the present paper is devoted to a more systematic investigation of this set for its own sake as well as its relation to the principal cluster set.

It is evident to begin with that both $\Pi(f, \zeta)$ and $\Pi_{*}(f, \zeta)$ are closed subsets of $\Omega$, and that

Received November 24, 1965.

* Research supported by the National Science Foundation. 


$$
\Pi(f, \zeta) \leqq \Pi_{x}(f, \zeta) \leqq C_{\mathscr{A}}(f, \zeta) \leqq C(f, \zeta) .
$$

Any condition, then, that guarantees that $\Pi(f, \zeta)=C_{\mathscr{A}}(f, \zeta)$ also ensures that $\Pi(f, \zeta)=\Pi_{\times}(f, \zeta)$, and several general conditions of this sort are known (see, e.g., $[10$, p. 389]).

Let $M$ be a closed subset of $\Omega$. Then Gross [11, p. 21] has shown that there exists a meromorphic function $f$ in $D$ and a point $\zeta \in \Gamma$ such that $\Pi(f, \zeta)=M$; there is even a holomorphic function with this property [11, p. $22]$.

We first show (Theorem 1) that this assertion is still valid if $\Pi(f, \zeta)$ is replaced by $\Pi_{x}(f, \zeta)$. Then we prove (Theorem 2) that if $N$ is a closed subset of $M$, there exists a meromorphic function $f(z)$ in $D$ for which $\Pi_{x}(f, \zeta)$ $=M$ and $\Pi(f, \zeta)=N$.

Suppose that there exist two chords, $X_{1}$ and $X_{2}$, at a point $\zeta \in \Gamma$ and a function $f(z)$ in $D$ for which

$$
C_{X_{1}}(f, \zeta) \cap C_{X_{2}}(f, \zeta)=\phi .
$$

Then we say that $\zeta$ is a chordally ambiguous point of $f$. We prove (Theorem $3)$ that given an enumerable subset $\dot{E}$ of $\Gamma$, there exists a bounded holomorphic function in $D$ for which each point of $E$ is a chordally ambiguous point of $f$. Each point $\zeta \in E$ then is a point for which $\Pi_{x}(f, \zeta)=\phi$, and $f$ is, in particular, a normal function (see [13]). We show (Theorem 4), however, that "most" points $\zeta \in \Gamma$, in a metrical as well as in a topological sense, are points at which $\Pi_{x}(f, \zeta) \neq \phi$ for a normal meromorphic function $f$. We prove (Theorems $5,6)$ that if, in addition, the set $A(f)$ is "small" in a metrical sense, and $f$ is not identically constant, then "most" points $\zeta \in \Gamma$ in a certain sense are such that actually $\Pi_{\times}(f, \zeta)=\Omega$. We also derive (Theorem 7 ) a sufficient condition for a normal holomorphic function $f$ in $D$ to satisfy $\Pi_{\mathrm{x}}(f, \zeta) \neq \phi$ at an individual point $\zeta \in \Gamma$.

The next three theorems (Theorems $8,9,10$ ) and corollaries thereto deal with the existence of functions $f$ with the property that $\Pi(f, \zeta)=\Pi_{x}(f, \zeta)=S$ for every $\zeta \in \Gamma$, where $S$ may be certain subsets of $\Omega$.

Define the exceptional chordal principal cluster set of $f$ at $\zeta \in \Gamma$ to be the set

$$
\Pi_{x}^{*}(f, \zeta)=\bigcap_{x} C_{x}(f, \zeta)
$$


where $X$ ranges over all but at most one of the chords at $\zeta$. This set is also of importance in Meier's work [12]. We prove (Theorem 11) a topological analogue (and a generalization) of a metrical theorem [5, p. 32, Theorem 6] involving $\Pi_{x}^{*}$.

Finally, some problems are posed in the Remarks scattered throughout the paper.

By a continuum we mean a nonempty, closed, connected subset of $\Omega$; it may consist of only a single point.

Lemma 1. Let $M$ be a closed subset of $\Omega$. Then there exists a sequence of continua $K_{n}(n=1,2,3, \ldots)$ such that $M=\bigcap_{n=1}^{\infty} K_{n}$.

Proof. The lemma is obviously true if $M=\phi$ or $M=\Omega$. Suppose that $M$ is a nonempty proper closed subset of $\Omega$. There is no loss of generality in assuming that $\infty \notin M$. Then there is a neighborhood $U$ of $\infty$ such that $M \cap \bar{U}$ $=\phi$, where $\bar{U}$ denotes the closure of $U$. Since the distance between $M$ and $\bar{U}$ is positive, if $\varepsilon_{1}>0$ is sufficiently small the closure of the open spherical cap with radius $\varepsilon_{1}$ about any point of $M$ as center lies in $\Omega-\bar{U}$. By the Heine-Borel theorem, finitely many of these spherical caps suffice to cover $M$; denote them by $D_{1}^{1}, D_{2}^{1}, \ldots, D_{n_{1}}^{1}$. We have

$$
M \subset \bigcup_{j=1}^{n_{1}} D_{j}^{1}
$$

and

$$
\bigcup_{j=1}^{n_{1}} \overline{D_{j}^{1}} \subset \Omega-\bar{U}
$$

Now the distance between $M$ and the frontier of $\bigcup_{j=1}^{n_{1}} D_{j}^{1}$ is positive, and so it is again possible to find a positive $\varepsilon_{2}<\frac{\varepsilon_{1}}{2}$ and a finite number of open spherical caps $D_{1}^{2}, D_{2}^{2}, \ldots, D_{n_{2}}^{2}$ with radii $\varepsilon_{2}$ about points of $M$ as centers such that

$$
M \subset \cup_{j=1}^{n_{2}} D_{j}^{2}
$$

and

$$
\bigcup_{j=1}^{n_{2}} \overline{D_{j}^{2}} \subset \bigcup_{j=1}^{n_{1}} D_{j}^{1}
$$

Repeating the argument we obtain for every $m>1$ a set of open spherical caps 
$D_{1}^{m}, D_{2}^{m}, \ldots, D_{n_{m}}^{m}$ with positive radii $\varepsilon_{m}<\frac{\varepsilon_{m-1}}{2}$ about points of $M$ as centers such that

$$
M \subset \bigcup_{j=1}^{n_{m}} D_{j}^{m}
$$

and

$$
\bigcup_{j=1}^{n m} \bar{D}_{j}^{m} \subset \bigcup_{j=1}^{n m-1} D_{j}^{m-1}
$$

For every natural number $m$, let $T_{m}=\bigcup_{j=1}^{n m} \bar{D}_{j}^{n}$. Then clearly

$$
T_{1} \supset T_{2} \supset \cdots \supset T_{m} \supset \cdots
$$

and

$$
M=\bigcap_{m=1}^{\infty} T_{m}
$$

Let $P_{1}, P_{2}, \ldots, P_{m}, \ldots$ be a sequence of parallels of latitude lying in $U$ and tending monotonically with $m$ to $\infty \in \Omega$. For each $j=1,2, \ldots, n_{1}$, let $L_{j}^{1}$ be an open arc of a circle of longitude such that $L_{j}^{1} \subset \Omega-T_{1}$ and $L_{j}^{1}$ extends from a point on the frontier of $D_{j}^{1}$ to a point of $P_{1}$ if that is possible, or to a point on the frontier of some $D_{k}^{1}$ with $k \neq j$ otherwise. Evidently the set

$$
T_{1} \cup\left(\bigcup_{j=1}^{n_{1}} L_{j}\right) \cup P_{1}
$$

is a continuum; call it $K_{1}$. Now for each $j=1,2, \ldots, n_{2}$ we define $L_{j}^{2}$ similarly, except that we use the sets $D_{j}^{2}$ and $P_{2}$ instead of $D_{j}^{1}$ and $P_{1}$, and further arrange it so that

$$
\left(\bigcup_{j=1}^{n_{2}} L_{j}^{2}\right) \cap\left(\bigcup_{j=1}^{n_{1}} L_{j}^{1}\right)=\phi
$$

which is evidently possible. The set

$$
T_{2} \cup\left(\bigcup_{j=1}^{n_{2}} L_{j}^{2}\right) \cup P_{2}
$$

is a continuum which we call $K_{2}$. Proceeding in this way by induction, we define the continua $K_{1}, K_{2}, \ldots, K_{m}, \ldots$ We claim that $M=\bigcap_{m=1}^{\infty} K_{m}$. How- 
ever, this is obvious in view of (1) and the facts that $\bigcap_{m=1}^{\infty} P_{m}=\phi$ and that

$$
\left(\bigcup_{j=1}^{n r} L_{j}^{r}\right) \cap\left(\bigcup_{j=1}^{n s} L_{j}^{s}\right)=\phi \quad(r \neq s)
$$

and the lemma is proved.

Theorem 1. Let $M$ be a closed subset of $\Omega$ and $\zeta$ be a point of $\Gamma$. Then there exists a holomorphic function $f(z)$ in $D$ such that $\Pi_{\times}(f, \zeta)=M$.

Proof. For convenience we may take $\zeta=1$. Denote by $B$ the circle $\left|z-\frac{1}{2}\right|=\frac{1}{2}$. Consider the enumerably many chords at $\zeta$ with initial points on $B$, which satisfy the conditions

$$
\arg (z-1)=\pi \pm \frac{n}{n+1} \frac{\pi}{2} \quad(n=0,1,2, \ldots),
$$

and arrange these chords in a sequence

$$
X_{1}, X_{2}, \ldots, X_{n}, \ldots
$$

Between every pair of chords (2) that are neighboring in the geometrical sense, we describe an arc $A$ at 1 , having no point in common with any chord (2), so as to oscillate between the pair of chords $X, X^{\prime}$ in question in such a way that any chord at 1 lying between $X$ and $X^{\prime}$ intersects $A$ in every neighborhood of the point 1 . Arrange the enumerably many arcs thus defined in a sequence

$$
\Lambda_{1}, \Lambda_{2}, \ldots, \Lambda_{n}, \ldots
$$

Let

$$
\left\{\omega_{1}, \omega_{2}, \ldots, \omega_{n}, \ldots\right\}
$$

be the set of complex numbers whose real and imaginary parts are both rational. For every $n$, define a continuous function $g_{2 n}(z)$ on $\Lambda_{n}$ in such a way that if the two neighboring chords (2) between which $A_{n}$ lies are $X$ and $X^{\prime}$, and if $X_{0}$ is any chord at 1 lying between $X$ and $X^{\prime}$, then for every natural number $k$, in every neighborhood of the point 1 the chord $X_{0}$ intersects the arc $A_{n}$ in a point at which the function $g_{2 n}(z)$ assumes the value $\omega_{k}$. According to Lemma 1 , there exists a sequence of continua $K_{1}, K_{2}, \ldots, K_{n}, \ldots$ such that $M=\bigcap_{n=1}^{\infty} K_{n}$. For every $n$, define a continuous function $g_{2 n-1}(z)$ on $X_{n}$ in such a way (see [7, p. 194]) that $C_{X_{n}}\left(g_{2 n-1}, 1\right)=K_{n}$. It follows from [7] that there exists a holo- 
morphic function $f(z)$ in $D$ such that, for every $n$,

$$
\lim _{\substack{|z| \rightarrow \mid \\ z \in \Lambda_{n}}}\left(f(z)-g_{2 n}(z)\right)=0, \quad \lim _{\substack{|z| \rightarrow \mid \\ z \in X_{n}}}\left(f(z)-g_{2 n-1}(z)\right)=0 .
$$

This implies that for every $n$,

$$
C_{X_{1}}(f, 1)=K_{n},
$$

whereas if $X$ is any chord at 1 not in the sequence (2), then

$$
C_{X}(f, 1)=\Omega \text {. }
$$

Evidently $\Pi_{x}(f, 1)=M$, which was to be proved.

Theorem 2. Let $M$ and $N$ be closed subsets of $\Omega$ with $M \geqq N$, and let $\zeta$ be a point of $\Gamma$. Then there exists a meromorphic function $f(z)$ in $D$ such that $\Pi_{\times}(f, \zeta)=M$ and $\Pi(f, \zeta)=N$.

Proof. It will be convenient again to take $\zeta=1$. According to Lemma 1 there are sequences of continua $K_{n}(n=1,2,3, \ldots)$ and $L_{n}(n=1,2,3, \ldots)$ such that

$$
M=\bigcap_{n=1}^{\infty} K_{n}
$$

and

$$
N=\bigcap_{n=1}^{\infty} L_{n}
$$

Define the circle $B$ and the sequences (2), (3) as in the proof of Theorem 1 . For $n=1,2,3, \ldots$, define $B_{z n-1}$ as the lower semicircular arc at 1 , and $B_{2 n}$ as the upper semicircular arc at 1 , of the circle of diameter $1+\frac{n}{n+1}$ that is internally tangent to $\Gamma$ at 1 . Define a continuous function $h_{n}(z)$ on $B_{n}$ in such a way $[7$, p. 194$]$ that

$$
C_{B_{n}}\left(h_{n}, 1\right)=L_{n} \quad(n=1,2,3, \ldots),
$$

and suppose that the functions $g_{n}(z)(n=1,2,3, \ldots)$ are defined as in the proof of Theorem 1. Denote by $\psi, z$ ) the resulting continuous function defined on $\left(\bigcup_{n=1}^{\infty} X_{n}\right) \cup\left(\bigcup_{n=1}^{\infty} A_{n}\right) \cup\left(\bigcup_{n=1}^{\infty} B_{n}\right)$.

It is readily seen that there is no loss of generality in assuming that $N$ does not contain $\infty$ as an isolated point (cf. $\left[2\right.$, p. 5]). Let $\nu_{n}(n=1,2,3, \ldots)$ 
be a sequence of complex numbers in $N$ that is everywhere dense in $N$ in the sense that every isolated point of $N$ occurs infinitely often as a term of the sequence. (In case $N=\phi$, the sequence $\left\{\nu_{n}\right\}$ is undefined and the rest of the construction is unnecessary.) Clearly there will be no harm in our assuming that the sequence $\left\{\omega_{n}\right\}$ has been redefined so as to contain the sequence $\left\{\nu_{n}\right\}$ as a subsequence, because the resulting sequence is still enumerable and everywhere dense on $\Omega$.

Consider $X_{1}, A_{1}$, and $B_{1}$. It follows from the definition of $\psi(z)$ that each of these arcs contains a point at which $\psi$ assumes the value $\nu_{1}$. Let $J_{1}$ be a Jordan curve in $D$ that contains these three points but contains no other point of any of the three arcs in question. Denote by $\delta_{1}$ the positive distance between $J_{1}$ and $\Gamma$, and by $X_{2}^{\prime}, A_{2}^{\prime}, B_{2}^{\prime}$ terminal subarcs of $X_{2}, A_{2}, B_{2}$ lying in the annulus $A_{1}: 1-\delta_{1}<|z|<1$. It follows again from the definition of $\psi(z)$ that each of the $\operatorname{arcs} X_{1}, \Lambda_{1}, B_{1}, X_{2}^{\prime}, A_{2}^{\prime}, B_{2}^{\prime}$ contains a point in the annulus $A_{1}$ at which $\psi$ assumes the value $\nu_{2}$. We let $J_{2}$ be a Jordan curve in $A_{1}$ that contains these six points but contains no other point of any of the six arcs in question. Denote by $\delta_{2}$ the positive distance between $J_{2}$ and $\Gamma$, and by $X_{3}^{\prime}, A_{3}^{\prime}, B_{3}^{\prime}$ terminal subarcs of $X_{3}, A_{3}, B_{3}$ lying in the annulus $A_{2}: 1-\delta_{2}<|z|<1$. We proceed in this fashion by induction, and then define the skeleton $S$ to be the set

$$
S=\left(X_{1} \cup \Lambda_{1} \cup B_{1}\right) \cup\left(\bigcup_{n=2}^{\infty}\left(X_{n}^{\prime} \cup A_{n}^{\prime} \cup B_{n}^{\prime}\right)\right) \cup\left(\bigcup_{n=1}^{\infty} J_{n}\right) .
$$

On $S$ we define the function $\varphi(z)$ to equal the constant $\nu_{n}$ everywhere on $J_{n}$ and to coincide with $\psi(z)$ at every other point of $S$. Then it is evident that $\varphi$ : is continuous on $S$. Consequently (cf. [2]) there exists a meromorphic function $f(z)$ in $D$ such that

$$
\lim _{\substack{|z| \rightarrow 1 \\ z \in \mathbb{S}}}|f(z)-\varphi(z)|=0
$$

This implies, in view of the proof of Theorem 1 , that $\Pi_{x}(f, 1)=M$; and (5) and (6) show that $\Pi(f, 1) \subseteq N$. Finally, (7) and the definition of $\varphi$ on the sequence $\left\{J_{n}\right\}$ make it clear that for every arc $\Xi$ at 1 ,

$$
C_{\Xi}(f, 1) \supseteqq N,
$$

and hence $\Pi(f, 1)=N$, which completes the proof.

Remark 1. It would be interesting to know whether Theorem 2 remains 
valid if the word "meromorphic" therein is replaced by "holomorphic". It is clear from the proof of Theorem 2 that this is so in case $N=\phi$.

THEOREM 3. Let $E$ be an enumerable subset of $\Gamma$. Then there exists a bounded holomorphic function $f(z)$ in $D$ such that every point of $E$ is a chordally ambiguous point of $f$.

Proof. Let $u(\zeta)$ be a bounded nonnegative real-valued function of $\zeta \in \Gamma$ such that, at every point $\zeta_{n} \in E$, the limits of $u(\zeta)$ as $\zeta$ approaches $\zeta_{n}$ from either side exist and are unequal (see, e.g., [14, p. 73]). Let $u(z)(z \in D)$ be the Poisson integral with respect to $u(\zeta)$. Then $[9, \mathrm{p} .62] u(z)$ is a bounded harmonic function in $D$, and $[15, \mathrm{p} .131]$ if $\zeta_{n} \in E, u(z)$ possesses distinct chordal limits on any two distinct chords at $\zeta_{n}$. Let $v(z)$ be a harmonic conjugate of $u(z)$ in $D$ and set

$$
f(z)=e^{u(z)+i v(z)}
$$

The function $f(z)$ then evidently has the desired properties.

Since the chordal principal cluster set is empty at a chordally ambiguous point, and since a bounded holomorphic function is normal, Theorem 3 implies the existence of normal holomorphic functions $f(z)$ in $D$ for which there exist points $\zeta \in \Gamma$ at which $\Pi_{\mathrm{x}}(f, \zeta)=\phi$. However, although there exists a normal holomorphic function $f(z)$ in $D$ such that $\Pi(f, \zeta)=\phi$ for every $\zeta \in \Gamma$ (see $[5$, p. 30 , Theorem 3$\rfloor$ ), in a metrical as well as in a topological sense there cannot be many points $\zeta \in \Gamma$ for a normal meromorphic function $f(z)$ in $D$ at which $\Pi_{x}(f, \zeta)=\phi$. This is shown by the following three theorems, which are immediate consequences of the Lemma, Theorem 2, and Theorem 1 in [3] if we observe that for the set $\Pi_{T}(f, \zeta)$ considered there we have always $\Pi_{T}(f, \zeta) \cong$ $\Pi_{\times}(f, \zeta)$.

THEOREM 4. If $f(z)$ is a normal meromorphic function in $D$, then there exists a residual subset $Q$ of $\Gamma$ of measure $2 \pi$ such that, for every $\zeta \in Q$, we have $\Pi_{x}(f, \zeta) \neq \phi$.

Remark 2. It would be interesting to know whether the requirement that the function be normal may be dropped in Theorem 4, at least for holomorphic functions (cf. [5, p. 32, Question 7]).

THEOREM 5. If $f(z)$ is a nonconstant normal meromorphic function in $D$ for 
SOME RESULTS AND PROBLEMS CONCERNING CHORDAL PRINCIPAL CLUSTER SETS 15

which $A(f)$ is of linear measure zero, then there exists a residual subset $R$ of $\Gamma$ such that, for every $\zeta \in R$, we have $\Pi_{x}(f, \zeta)=\Omega$.

THEOREM 6. If $f(z)$ is a nonconstant normal meromorphic function in $D$ for which $A(f)$ is of harmonic measure zero, then there exists a residual subset $S$ of $\Gamma$ of measure $2 \pi$ such that, for every $\zeta \in S$, we have $\Pi_{\times}(f, \zeta)=\Omega$.

THEOREM 7. Let $f(z)$ be a normal holomorphic function in $D$, and suppose that $\zeta \in \Gamma$. If $f$ is unbounded in some Stolz angle $\Delta$ at $\zeta$, then $\Pi_{x}(f, \zeta) \neq \phi$.

Proof. If $X$ is any chord at $\zeta$, then $\infty \in C_{X}(f, \zeta)$, otherwise $[4$, p. 402 , Theorem 4] $f$ would be bounded on $\Delta$. Hence, $\infty \in \Pi_{x}(f, \zeta)$.

Remark 3. Let $\mu(z)$ denote the elliptic modular function in $D$. If $\zeta \in \Gamma$ and if $\zeta$ is not one of the enumerably many vertices on $\Gamma$ of the modular figure, is it true that $\mu$ is unbounded in some Stolz angle at $\zeta$ ? If so, it would follow from Theorem 7 that, for every $\zeta \in \Gamma$, we have $\Pi_{x}(\mu, \zeta)-\Pi(\mu, \zeta) \neq \phi$, which would answer [5, p. 33, Question 8].

THEOREM 8. There exists a holomorphic function $f(z)$ in $D$ such that $\Pi(f, \zeta)$ $=\Pi_{\times}(f, \zeta)=\Omega$ for every $\zeta \in \Gamma$.

Proof. This theorem is an immediate consequence of [6, p. 1255, Corollary 2].

ThEOREM 9. There exists a nonconstant holomorphic function $f(z)$ in $D$ such that $\Pi(f, \zeta)=\Pi_{x}(f, \zeta)=\{\infty\}$ for every $\zeta \in \Gamma$.

Proof. Consider the holomorphic function $f(z)$ constructed in $[8$, Theorem 1]. For every $\zeta \in \Gamma$, it is evident that (i) $\infty \in C_{X}(f, \zeta)$ for every chord $X$ at $\zeta$, (ii) that $\infty \in C_{\Delta}(f, \zeta)$ for every arc $A$ at $\zeta$, and (iii) that $\left\{\infty_{\infty}\right\}=C_{X^{\prime}}(f, \zeta)$ for some chord $X^{\prime}$ at $\zeta$; and the conclusion of the theorem follows.

Corollary 1. Let $\omega \in \Omega$. Then there exists a nonconstant meromorphic function $f(z)$ in $D$ such that $\Pi(f, \zeta)=\Pi_{x}(f, \zeta)=\{\omega\}$ for every $\zeta \in I$ :

Proof. Denote by $g(z)$ the function considered in Theorem 9. If $\omega=\infty$, let $f(z) \equiv g(z)$; if $\omega=0$, let $f(z) \equiv \frac{1}{g(z)}$; if $\omega \neq 0, \infty$, let $f(z) \equiv \frac{1}{g(z)}+\omega$.

Remark 4. In view of Theorem 2, the following problem is suggested by Corollary 1 and Theorem 8 : Let $M$ be a nonempty closed subset of $\Omega$. Does 
there exist a meromorphic function $f(z)$ in $D$ such that $\Pi(f, \zeta)=\Pi_{x}(f, \zeta)=M$ for every $\zeta \in \Gamma$ ? Cf. Remark 5 .

ThEOREM 10. There exists a nonconstant holomorphic function $f(z)$ in $D$ such that $\Pi_{\times}(f, \zeta)=\{0\}$ for every $\zeta \in \Gamma$.

Proof. Let $K$ be the circle $|z|=\frac{1}{2}$, and consider the set $S$ of all points on the circle $K$ of the form

$$
z=\frac{1}{2} e^{ \pm i\left(0 . t_{1} t_{2} t_{3} \ldots\right) \pi}
$$

where $0 . t_{1} t_{2} t_{3} \ldots$ is a ternary fraction in which each $t_{j}$ is either 0 or 2 . For every $z \in S$, let $X_{z}$ be the chord extending from the point $z$ to the point

$$
\zeta=e^{ \pm i\left(0 . b_{1} b_{2} b_{3} \cdots\right) \pi} \in \Gamma
$$

where $0 . b_{1} b_{2} b_{3} \ldots$ is the binary fraction such that, for $j=1,2,3, \ldots$,

$$
b_{j}=\left\{\begin{array}{lll}
0 & \text { if } & t_{j}=0, \\
1 & \text { if } & t_{j}=2 .
\end{array}\right.
$$

The set $S$ is a perfect nowhere dense subset of $K$. Let $A_{1}, A_{2}, A_{3}, \ldots$ be the enumerably many open subarcs of $K$ that are complementary to $S$. Denote by $z_{m 1}, z_{m 2}$ the left and right end points of $A_{m}$ as viewed by an observer at the origin, and let $\mu_{m}$ be the midpoint of the arc $A_{m}$.

To every point $\zeta$ of $\Gamma$, with the exception of an enumerable everywhere dense subset $V$ of $\Gamma$, there corresponds exactly one $z \in S$ such that $X_{z}$ is a chord at $\zeta$.

On the other hand, to every point $\zeta \in V$ there correspond exactly two points, $z_{m 1}, z_{m 2}$, in $S$ such that $X_{z_{m 1}}$ and $X_{z_{m 2}}$ are chords at $\zeta$. The region whose boundary is

$$
A_{m} \cup X_{z_{m 1}} \cup X_{z_{m 2}} \cup\{\zeta\}
$$

will be called $\Delta_{\zeta}$. Let $J_{m}$ be an arc at $\zeta$ whose initial point is $\mu_{m}$ and which, except for $\mu_{m}$, lies in $\Delta_{j}$; and let $J_{m}$ oscillate between $X_{z_{m 1}}$ and $X_{z_{m}}$ in such a way that every chord at $\zeta$ that intersects $A_{m}$ also intersects $J_{m}$ infinitely often in every neighborhood of $\zeta$. Also, for some $\zeta \in V$, let $J_{0}$ be an arc at $\zeta$ that lies in $A_{\zeta}$ but does not intersect any $J_{m}(m \geqq 1)$. We may choose each $J_{m}$ ( $m \geqq 0$ ) in such a manner that as $z \rightarrow \zeta$ on $J_{m},|z| \rightarrow 1$ monotonically. 
We now define a function $g(z)$. For every $z \in S$, let $g(z) \equiv 0$ on $X z$. For $m=1,2,3, \ldots$, define $g(z) \equiv 0$ on $J_{m}$. Finally, let $g(z) \equiv 1$ on $J_{0}$.

Denote by $T$ the set of points on which $g(z)$ has thus been defined. Then it follows from the proof of $[7$, p. 190, Corollary 2] that there exists a holomorphic function $f(z)$ in $D$ such that

$$
\lim _{\substack{|z| \rightarrow \mid \\ z \in \mathbb{T}}}(f(z)-g(z))=0
$$

uniformly. Clearly then, for every $\zeta \in \Gamma$, there exists at least one chord at $\zeta$ on which $f(z) \rightarrow 0$ as $z \rightarrow \zeta$. Furthermore, it is readily seen that $0 \in C_{X}(f, \zeta)$ for every chord $X$ at $\zeta$. Hence, $\Pi_{x}(f, \zeta)=\{0\}$. Finally, in view of the definition of $g(z)$ on $J_{0}, f(z)$ is not identically constant.

Corollary 2. Let $\omega \in \Omega$. Then there exists a nonconstant holomorphic function $f(z)$ in $D$ such that $\Pi_{x}(f, \zeta)=\{\omega\}$ for every $\zeta \in \Gamma$.

Proof. This follows from Theorems 9 and 10.

Remark 5. Does there exist a nonconstant holomorphic (or meromorphic) function $f(z)$ in $D$ such that $\Pi_{x}(f, \zeta)=\phi$ for every $\zeta \in \Gamma$ ? This is related to the following question: If $f(z)$ is holomorphic (or meromorphic) in $D$, is it true that $\Pi_{x}(f, \zeta) \neq \phi$ at almost every or at nearly every Plessner point of $f$ ? (By almost every (nearly every) point of $\Gamma$ we mean every point of $\Gamma$ with the exception of a set of Lebesgue measure zero (first Baire category).) If the answer to the second question is positive, then Plessner's theorem or Meier's theorem [12, p. 330, Theorem 5] shows that the answer to the first question is negative. Cf. Remark 2 .

Corollary 3. Let $\omega$ be a finite complex number. Then there exists a nonconstant holomorphic function $f(z)$ in $D$ such that, for every $\zeta \in \Gamma$ with at most enumerably many exceptions, $\Pi(f, \zeta)=\Pi_{x}(f, \zeta)=\{\omega\}$.

Proof. In view of the proof of Theorem 10, there exists a nonconstant holomorphic function $f(z)$ in $D$ such that, for every $\zeta \in \Gamma, \Pi_{\times}(f, \zeta)=\{\omega\}$ and there exists at least one chord $X$ at $\zeta$ with $C_{X}(f, \zeta)=\{\omega\}$. Corollary 3 now follows from the ambiguous-point theorem [1, p. 380, Theorem 2].

Remark 6. Does Corollary 3 remain valid if the phrase "with at most enumerably many exceptions" is deleted? 
TheOREM 11. Let $f(z)$ be a holomorphic function in $D$. Then $\Pi_{x}^{*}(f, \zeta) \neq \phi$ for nearly every $\zeta \doteq \Gamma$.

Proof. According to [12, p. 330, Theorem 8], for nearly every point $\zeta \in \Gamma$, either $\Pi_{\times}(f, \zeta)=C(f, \zeta)$ or there is at most one chord at $\zeta$ on which $f$ is bounded. Hence, since $C(f, \zeta) \neq \phi$, either $\Pi_{x}(f, \zeta) \neq \phi$, which implies that $\Pi_{x}^{*}(f, \zeta) \neq \phi$, or else $\infty \in \Pi_{x}^{*}(f, \zeta)$, which again means that $\Pi_{x}^{*}(f, \zeta) \neq \phi$.

Corollary 4. Let $f(z)$ be a holomorphic function in $D$. Then $\Pi_{x}^{*}(f, \zeta) \neq \phi$ for almost every and nearly every $\zeta \in \Gamma$.

Proof. This is an immediate consequence of Theorem 11 and $[5$, p. 32 , Theorem 6].

\section{REFERENCES}

[1] F. Bagemihl, Curvilinear cluster sets of arbitrary functions, Proc. Nat. Acad. Sci. 41 (1955), 379-382.

[2] - Characterization of the set of values approached by a meromorphic function on sequences of Jordan curves, Ann. Acad. Sci. Fennicæ A I 328 (1963), 1-14.

[ 3 ] - Some approximation theorems for normal functions, Ann. Acad. Sci. Fennicæ A I 335 (1963), 1-5.

[4] - , Some boundary properties of normal functions bounded on nontangential arcs, Archiv der Math. 14 (1963), 399-406.

[5] - G. Piranian and G. S. Young, Intersections of cluster sets, Bul. Inst. Politehn. Iasi (N. S.) 5 (1959), 29-34.

[6] - and W. Seidel, Spiral and other asymptotic paths, and paths of complete indetermination, of analytic and meromorphic functions, Proc. Nat. Acad. Sci. 39 (1953), $1251-1258$.

[7] - and - Some boundary properties of analytic functions, Math. Zeitschr. 61 (1954), 186-199.

[8] _ and - Some remarks on boundary behavior of analytic and meromorphic functions, Nagoya Math. J. 9 (1955), 79-85.

[ 9 ] L. Bieberbach, Lehrbuch der Funktionentheorie (2d ed.) vol. 2, Leipzig, 1931.

[10] E. F. Collingwood, On sets of maximum indetermination of analytic functions, Math. Zeitschr. 67 (1957), 377-396.

[11] W. Gross, Über die Singularitäten analytischer Funktionen, Mh. Math. Phys. 29 (1918), 3-47.

[12] K. Meier, Über die Randwerte der meromorphen Funktionen, Math. Ann. 142 (1961), 328-344.

[13] K. Noshiro, Cluster sets, Berlin, 1960.

[14] W. Rudin, Principles of mathematical analysis, New York, 1953.

[15] M. Tsuji, Potential theory in modern function theory, Tokyo, 1959.

\section{University of Wisconsin-Milwaukee}

